\title{
An Energy Segmentation Method of High-resolution SAR Image Based on Multiple Features
}

\author{
Wang Yu*, You Haotian, Liu Taolin \\ College of Geomatics and Geoinformation, Guilin University of Technology, Guilin, Guangxi 541004, China
}

\section{Commission}

KEY WORDS: High-resolution SAR Image Segmentation; Multiple Features; Energy Function; Regular Tessellation; RJMCMC Algorithm

\begin{abstract}
:
To achieve the optimal image segmentation, an energy segmentation method based on multiple features and blocks for high resolution Synthetic Aperture Radar (SAR) image is proposed in this paper. First of all, a feature vector of pixel is formed with the texture feature extracted by curvelet transform and means function, the boundary feature extracted by curvelet transform and Canny Operator, and the original spectral feature; a feature set is formed by all feature vectors of pixels in the image. The feature vector is considered as segmentation basis, and its domain is partitioned by regular tessellation. On the partitioned image domain, a label variable is assigned to a regular block; each homogeneous region is fitted by one or more regular blocks; Obviously, a label field is formed by all the label variables of regular blocks. The model of label field is built by using energy function of neighborhood relationship. The feature set is considered as a realization of a random filed of multiple features (multiple features field for short). A heterogeneous energy function is used to establish the model of multiple features field. Then the established models of the label field and multiple features field are combined to define global energy function of image segmentation, and non-constrained Gibbs probability distribution is used to describe the global energy function to build the energy segmentation model based on multiple features. Further, a RJMCMC algorithm is designed to simulate from the model to segment SAR image. To verify the feasibility and superiority of the proposed approach, real SAR images are tested.
\end{abstract}

\section{INTRODUCTION}

Image segmentation is a procedure of partitioning a digital image into some different meaningful regions with homogeneous characteristics (Hehmati et al., 2016). Synthetic Aperture Radar (SAR) image contains rich features (such as spectral, texture, boundary features), they are very important for image segmentation. However, traditional SAR image segmentation method based on spectral feature can not segment SAR image well. The reasons derive from the inherent speckle noise of SAR image, largely differences between homogeneous regions, and small differences between heterogeneous regions. In order to improve the accuracy of image segmentation, many segmentation methods based on multiple features are proposed (Muneeswaran et al., 2006; Yang et al., 2014; Patel et al., 2011; Li et al., 2015). These methods use the advantages of different image features to segment image well.

With the development of multiresolution analysis, the image procedure methods based on multiresolution analysis are proposed (Chang and Kuo, 1993; Jung, 2007; Zheng et al., 2012). The proposed methods use multiresolution analysis to extract multiple features, and bring them into image segmentation. Wan et al. (2011) presents a segmentation method of noise image combined wavelet transform and Cauchy density. The proposed method is effective for noise image corrupted by artificial or natural noise, but the wavelet transform does not work appropriately in capturing the detail and boundary information of image, it leads to over-segmentation. To solve the over-segmentation in the wavelet domain, Li et al. (2006) proposed an approximate finite redgelet transform algorithm for SAR image segmentation, but the finite redgelet transform does not work appropriately in capturing the curves, it leads to low accuracy of boundary segmentation. Curvelet transform is a newest extension of wavelet and ridgelet transform which focus on compacting with exciting experiences taking place along curves, so it can extract image features well. However, the research of image processing

*Corresponding author: Yu Wang; Email address: 1009059221@qq.com 
based on curvelet transform is not perfect.

To achieve the optimal SAR image segmentation, an energy segmentation method based on multiple features and blocks for high resolution SAR image is proposed. First of all, texture and boundary features are extracted by curvelet transform, and they and the original spectral feature are used to form a feature set of SAR image. The feature vector is considered as segmentation basis, and its domain is partitioned by regular tessellation. On the partitioned image domain, the global potential energy function and non-constrained Gibbs probability distribution are used to build the energy segmentation model based on multiple features. Further, a RJMCMC algorithm is designed to simulate from the model to segment SAR image. Real SAR images are tested, and the segmentation results are analyzed; the qualitative and quantitative experiment results show the feasibility and superiority of the proposed approach.

The rest of this paper is organized as follows. In Section 2 we present the proposed algorithm. We then in Section 3 detail and discuss the results of real SAR images. Finally, Section 4 contains conclusions and perspectives for further research.

\section{DESCRIPTION OF PROPOSED ALGORITHM}

\subsection{Curvelet Transform}

Candes et al (2006) described two digital implementations of a novel mathematical transform, namely, the second generation curvelet transform. This paper uses it, which is based on the wrapping of specifically chosen Fourier samples. The steps are as followed (Candes et al., 2006):

1) Apply the 2D Fast Fourier Transform (FFT) to an image to obtain Fourier samples $\hat{f}\left[n_{1}, n_{2}\right]$;

2) For each scale $j$ and angle $l$, form the product $\tilde{U}_{j}\left[n_{1}, n_{2}\right] \tilde{f}_{j, l}\left[n_{1}, n_{2}\right]$;

3) Wrapping the product around the origin and obtain

$$
\tilde{f}_{j, l}\left[n_{1}, n_{2}\right]=W\left(\tilde{U}_{j}, \tilde{f}_{j, l}\right)\left[n_{1}, n_{2}\right]
$$

4) Apply the inverse $2 \mathrm{D}$ FFT to each $\tilde{f}_{j, l}$, hence collecting the discrete coefficients $C^{D}(j, l, k)$.

\subsection{The Proposed Algorithm}

Consider a SAR intensity image $\boldsymbol{z}=\left\{z_{d}, d \in \boldsymbol{D}\right\}$, where $d$ is the index of pixel, $z_{d}$ is the intensity of pixel $d, \boldsymbol{D}$ is image domain.

Spectral, texture and boundary features are three basic features of SAR image, there are extracted and used to form a feature set in this paper, where spectral feature is select the original intensity feature, that is, $\boldsymbol{z}=\left\{z_{d}, d \in \boldsymbol{D}\right\}$; texture and boundary features are used curvelet coefficients obtained by curvelet transform.

The operation of extracting texture feature is as follows. First, the curvelet transform is used to decompose a sub-image with $c_{t} \times c_{t}$ pixels centered on pixel $s$ to obtain a series of curvelet coefficients. Then their means is computed as

$$
\mu_{s}=\frac{1}{N_{t}} \sum_{j, l, k}\left|C^{D}(j, l, k)\right|
$$

where $N_{t}$ is the total number of curvelet coefficients. In a ccordance with the above procedure, the t-exture feature of the SAR image is obtained, that is, $\mu=\left\{\mu_{s} ; s=1, \ldots\right.$, $S\}$.

The operation of extracting boundary feature is as follows. Firstly, the curvelet transform is used to decompose the SAR image $\boldsymbol{f}$ to obtain a series of curvelet coefficients. Then, a Canny operator is just used for extracting the boundary of curvelet coefficients on the coarse and finest scales, their non-boundary curvelet coefficients are changed to 0 , and the detail coefficients are unchanged. Finally, all the curvelet coefficients are reconstructed to obtain the boundary feature of the SAR image $\boldsymbol{b}$ $=\left\{b_{s} ; s=1, \ldots, S\right\}$,

$$
\boldsymbol{b}=\sum_{j, l, k} C^{D}(j, l, k) \phi_{j, l, k}^{D}
$$

In summary, the feature set can be written as $\boldsymbol{f}=\{\boldsymbol{z}, \boldsymbol{u}, \boldsymbol{b}\}=$ $\left\{\boldsymbol{f}_{s}, s=1, \ldots, S\right\}$, where $\boldsymbol{f}_{s}=\left\{f_{g s}, g=1, \ldots, G\right\}$ is the feature vector values of pixel $s, g$ is feature index.

As the effect of speckle noise in the SAR image, the traditional segmentation method based on pixel can not segment well in the homogeneous regions. To improve the segmentation accuracy in the homogeneous regions, the sub-region is considered as a processing unit, that is, an image domain is partitioned by the geometric partitioning technique, and region-based statistic models are built on the partitioned image domain. In this paper, regular tessellation is used to partition $\boldsymbol{D}$ into $I$ regular blocks, so $\boldsymbol{D}$ can be rewritten as $\boldsymbol{D}=$ $\left\{P_{i}, i=1, \ldots, I\right\}$, where $I$ is unknown, $N$ is the total number of pixels in the SAR image $\boldsymbol{z}$.

Let label field $\boldsymbol{L}=\left\{L_{i}, i=1, \ldots, I\right\}$, where $L_{i} \in\{1, \ldots, k\}$ is the label variable of block, $k$ is the number of classes and is known. $\boldsymbol{l}$ is a realization of $\boldsymbol{L} . \boldsymbol{l}$ is defined by potential energy, 


$$
U_{l}(\boldsymbol{l})=\sum_{i=1}^{I}\left\{-\eta\left[\sum_{P_{i^{\prime}} \in N P_{i}} 2 \delta\left(l_{i}, l_{i^{\prime}}\right)-1\right]\right\}
$$

where $\eta$ is spatial interaction parameter, $N P_{i}$ is the set of the neighbor blocks of $P_{i} ; l_{i}=l_{i^{\prime}}$, if $\delta\left(l_{i}, l_{i^{\prime}}\right)=1$, otherwise, $\delta\left(l_{i}, l_{i^{\prime}}\right)$ $=0$.

Then $U_{f}(f, l)$, represents the relationship between the characteristic field $\boldsymbol{F}$ and label field $\boldsymbol{L}$, is defined using the sum of heterogenous potential energy function,

$$
U_{f}(\boldsymbol{f}, \boldsymbol{l})=\sum_{s=1}^{S} \sum_{i=1}^{I} V\left(\boldsymbol{f}_{s i}, \boldsymbol{f}_{s t}\right)
$$

where, $\boldsymbol{f}_{s t}=\left\{\boldsymbol{f}_{s i} ; l_{i}=t\right\}, V\left(\boldsymbol{f}_{s i}, \boldsymbol{f}_{s t}\right)$, represents the heterogenous potential energy function, is defined by K-S distance in multiscale texture feature images (Kervrrann and Heitz, 1995)

$$
\begin{aligned}
& V\left(\boldsymbol{f}_{s i}, \boldsymbol{f}_{s t}\right)=d_{K S}\left(\boldsymbol{f}_{s i}, \boldsymbol{f}_{s t}\right) \\
& =\max _{-\infty<z<+\infty}\left|\hat{F}_{f_{s i}}(z)-\hat{F}_{f_{s t}}(z)\right|
\end{aligned}
$$

where $\hat{F}_{f_{s i}}$ and $\hat{F}_{f_{s t}}$ are cumulative sampling distribution of $\boldsymbol{f}_{s i}$ and $\boldsymbol{f}_{\text {st. }} d_{K S}$ is K-S distance, that is, the maximum distance between $\hat{F}_{f_{s i}}$ and $\hat{F}_{f_{s t}}$.

Combining (3)-(5), the global potential energy function of image segmentation, can be defined on the partitioned domain

$$
U(\boldsymbol{f}, \boldsymbol{l})=U_{l}(\boldsymbol{l})+U_{f}(\boldsymbol{f}, \boldsymbol{l})
$$

The non-constrained Gibbs probability distribution is used to describe the global energy function of the image segmentation model based on region and multiscale features

$$
\begin{aligned}
& G(\boldsymbol{f}, \boldsymbol{l})=\frac{1}{A} \exp \{-U(\boldsymbol{f}, \boldsymbol{l})\} \\
& =\frac{1}{A} \exp \sum_{i=1}^{I}\left\{\eta\left[\sum_{P_{i^{\prime}} \in N P_{i}} 2 \delta\left(l_{i}, l_{i^{\prime}}\right)-1\right]\right\} \\
& +\exp \left\{-\sum_{s=1}^{S} \sum_{i=1}^{I} d_{K S}\left(\boldsymbol{f}_{s i}, \boldsymbol{f}_{s t}\right)\right\}
\end{aligned}
$$

In order to achieve the optimal image segmentation, a RJMCMC algorithm is designed to simulate the segmentation model. In the processing of simulation, two moves are designed, involving updating label field, and splitting or merging the blocks.

1) Updating label field: the specific operation of the move is: a block $P_{i}$ is randomly chosen, proposing a new real label $l_{i}^{*}$ in $\{1, \ldots, k\}\left(l_{i}^{*} \neq l_{i}\right)$ and performing a switch if the proposal is accepted. The acceptance probability can be written as
(Green, 1995)

$$
a_{t}=\min \left\{1, R_{t}\right\}
$$

where

$$
R_{t}=\frac{U_{l}\left(\boldsymbol{l}^{*}\right)+U_{f}\left(\boldsymbol{f}, \boldsymbol{l}^{*}\right)}{U_{l}(\boldsymbol{l})+U_{f}(\boldsymbol{f}, \boldsymbol{l})}
$$

where $\boldsymbol{l}^{*}=\left\{l_{1}, \ldots, l_{i}^{*}, \ldots, l_{I}\right\}, \boldsymbol{l}=\left\{l_{1}, \ldots, l_{i}, \ldots, l_{I}\right\}$.

2) Splitting or merging blocks: The move of splitting block is in tandem with the move of merging blocks. The splitting move is an operation that a block is split to two blocks with different class labels, and The new partition of image domain becomes $\boldsymbol{D}^{*}=\left\{P_{1}, \ldots, P_{i}^{*}, \ldots, P_{I}, P_{I+1}^{*}\right\}$, the acceptance probability of the splitting move can be expressed as,

$$
a_{f_{I}}\left(\boldsymbol{D}, \boldsymbol{D}^{*}\right)=\min \left\{1, R_{f_{I}}\right\}
$$

where

$$
R_{f_{I}}=\frac{U_{l}\left(\boldsymbol{l}^{*}\right)+U_{f}\left(\boldsymbol{f}, \boldsymbol{l}^{*}\right)}{U_{l}(\boldsymbol{l})+U_{f}(\boldsymbol{f}, \boldsymbol{l})} \times \frac{r_{h_{I+1}}\left(\boldsymbol{\Theta}^{*}\right)}{r_{f_{I}}(\boldsymbol{\Theta}) q(\boldsymbol{u})}\left|\frac{\partial \boldsymbol{\Theta}^{*}}{\partial(\boldsymbol{\Theta}, \boldsymbol{u})}\right|
$$

where $r_{f_{I}}=f_{I} / I, \quad r_{h_{I+1}}=h_{I+1} /(I+1), \quad f_{I}$ and $h_{I+1}$ are the probabilities of choosing the split and merge proposal, respectively; $\Theta^{*}=\left(\boldsymbol{l}^{*}, I+1\right), \Theta=(\boldsymbol{l}, I) ; \boldsymbol{l}^{*}=\left\{l_{1}, \ldots, l_{i}^{*}, \ldots, l_{I}\right.$, $\left.l_{I+1}{ }^{*}\right\}, \boldsymbol{l}=\left\{l_{1}, \ldots, l_{i}, \ldots, l_{I}\right\} ; \boldsymbol{u}=l_{I+1}{ }^{*}$; The Jacobian term in Eq. (11) is equal to 1

\section{EXPERIMENTAL RESULTS AND DISCUSSION}

To illustrate the feasibility and superiority of the proposed approach, two real SAR images with $128 \times 128$ pixels shown in Figure 1 are tested.

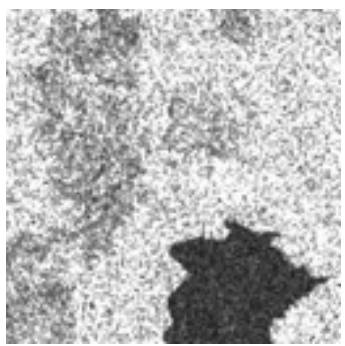

(a)

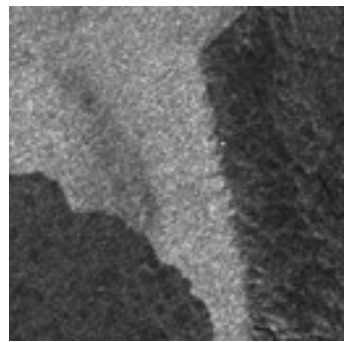

(b)
Figure 1. Real SAR images: (a) coastal image, and (b) city image.

Figure 2 show the segmentation results of two real SAR images. From Figure 2, it can be found that the proposed method can segment homogenous regions well. In order to qualitatively evaluate the segmentation results, overlaying the 
outlines on the original images shown in Figure 3. From Figure 3 , it can be seen that the extracted outlines of segmentation results match the real outlines well.

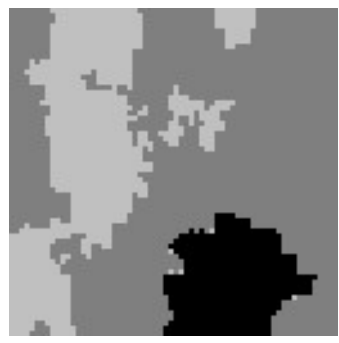

(a)

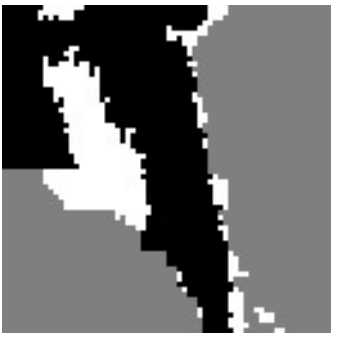

(b)
Figure 2. Segmentation results: (a) coastal image segmentation, and (b) city image segmentation.

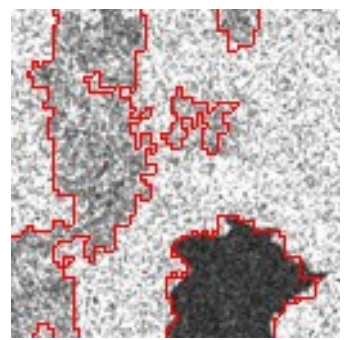

(a)

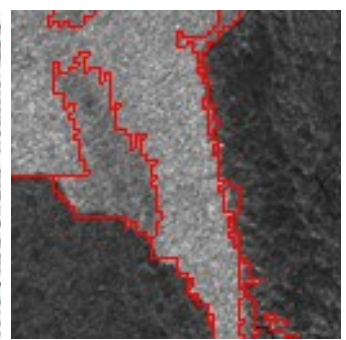

(b)
Figure 3. Visual evaluation: (a)-(b) overlaying the outlines on the original images

In order to assess the proposed method quantitatively, some common measures, including producer's accuracy, user's accuracy, overall accuracy and kappa coefficient, are computer based on the simulated real SAR images in Figure 4. Table 1 lists them. As is shown in Table 1, it can be seen that the overall accuracies are greater than or equal to $93.7 \%$. In addition, the kappa coefficients are up to 0.900 . It can be illustrated that the proposed approach is feasible and effective.

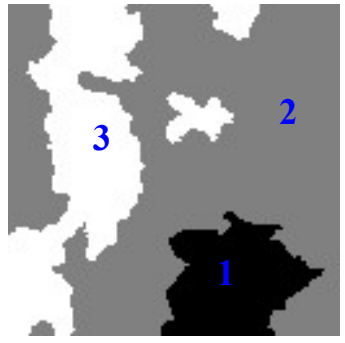

(a)

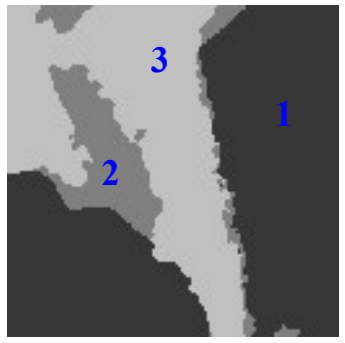

(b)
Figure 4. Templates of real SAR images: (a) coastal image template, and (b) city image template.

Table 1. Quantitative evaluation

\begin{tabular}{cccc}
\hline image & & $\mathrm{a}$ & $\mathrm{b}$ \\
\hline \multirow{2}{*}{ producer's accuracy } & $C_{1}$ & 95.5 & 98.2 \\
$(\%)$ & $C_{2}$ & 93.7 & 90.6 \\
& $C_{3}$ & 92.8 & 91.5 \\
\hline
\end{tabular}

\begin{tabular}{lccc}
\hline & $C_{1}$ & 93.2 & 97.8 \\
user's accuracy (\%) & $C_{2}$ & 96.2 & 83.9 \\
& $C_{3}$ & 90.2 & 95.6 \\
overall accuracy (\%) & & 93.7 & 94.0 \\
kappa coefficient & & 0.902 & 0.906 \\
\hline
\end{tabular}

\section{CONCLUSIONS}

This paper presents a multiple feature- and block-based energy segmentation method for high-resolution SAR image. The proposed method effectively uses curvelet transform and statistic method to obtain multiple features, and use it to overcome speckle noise to segment SAR image well, but the number of classes is given by user. In the future, it is a research topic to determine the number automatically.

\section{REFERENCES}

Candes, E., Demanet, L., Donoho, D, et al. 2006. Fast discrete Curvelet Transform. Multiscale Modeling \& Simulation, 5(3): 861-899.

Chang, T., Kuo, J. C. C. 1993. Texture analysis and classification with Tree-Structured Wavelet Transform. IEEE Transaction and Image Processing, 2(4): 429-441.

Green, P. J. 1995. Reversible jump Markov chain Monte Carlo computation and Bayesian model determination. Biometrika, 82(4): 711-732.

Heshmati, A., Gholami, M., Rashno, A. 2016. Scheme for unsupervised colour-texture image segmentation using neutrosophic set and non-subsampled contourlet transform. IET Image Process, 10(6): 464-473.

Jung, C. R. 2007. Combining wavelets and watersheds for robust multiscale image segmentation. Image and Vision Computing, 25(1): 24-33.

Kervrrann, C., Heitz, F. 1995. A markov random field model-based approach to unsupervised texture segmentation using local and global spatial statistics. IEEE Transactions on Image Processing, 4(6): 856-862.

Li, J. Y., Zhang, H. Y., Zhang, L. P. 2015. A nonlinear multiple feature learning classifier for hyperspectral images with limited training samples. IEEE Journal of Selected Topics in Applied Earth Observations and Remote Sensing, 8(6): 1-11.

Li, Y. Q., He, M. Y., Li, C. X. 2006. An approximate finite 
ridgelet transform algorithm for SAR image segmentation.

2006 8th international Conference on Signal Processing, Gulin, China, pp. 1-4.

Muneeswaran, K., Ganesan, L., Arumumgam, S., et al. 2006. Texture image segmentation using combined features from spatial and spectral distribution. Pattern Recognition Letters, 27(7): 755-764

Patel, H. N., Jain, R. K., Joshi, M. V. 2011. Fruit detection using improved multiple features based algorithm. International Journal of Computer Applications, 13(2): 1-5.

Wan, T., Canagarajah, N. M., Achim, A. 2011. Segmentation of noise colour images using Cauchy distribution in the complex wavelet domain. IET Image Process, 5(2):159-170.
Yang, H. Y., Zhang, X. J., Wang, X. Y. 2014. LS-SVM-based image segmentation using pixel color-texture descriptors. Pattern Analysis Applications, 17(2): 341-359.

Zheng, C.,Qin, Q., Liu., G., et al. 2012. Image segmentation based on multiresolution Markov random field with fuzzy constraint in wavelet domain. IET Image Process, 6(3): 213-221. 